\title{
Multi-Frequency High-Field EPR Study of Iron Centers in Malarial Pigments
}

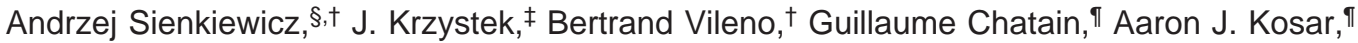 \\ D. Scott Bohle, ${ }^{\star, \oplus}$ and Lászlo Forro ${ }^{\dagger}$ \\ Institute of Physics, Polish Academy of Sciences, Al. Lotników 32/46, 02-668 Warsaw, Poland, Institute of Physics \\ of Complex Matter, Ecole Polytechnique Fédérale, CH-1015 Lausanne, Switzerland, National High Magnetic Field \\ Laboratory, Florida State University, 1800 East Paul Dirac Drive, Tallahassee, Florida 32310, Department of \\ Chemistry, McGill University, 801 Sherbrooke Street West, Montreal, Quebec H3A 2K6, Canada
}

Received January 13, 2006; E-mail: scott.bohle@mcgill.ca

Malaria, whose most severe form is caused by a protozoan parasite, Plasmodium falciparum $(P f)$, remains the world's most prevalent vector-borne disease. The spread of chloroquine-resistant strains $(\mathrm{CQR})$ of $P f$ and the absence of a suitable replacement for this once effective antimalarial created an urgent need to understand the biochemistry behind the drug's action. ${ }^{1}$ The intraerythrocytic growth stage of $P f$ involves hemoglobin proteolysis as the primary nutrient source with the concomitant release of free heme. The liberated heme is detoxified by the parasite into an inert crystalline material, called malarial pigment, or hemozoin..$^{2,3}$ According to the recent hypothesis, chloroquine inhibits heme aggregation in ring or early-stage malaria trophozoites. ${ }^{4}$ It has been shown that hemozoin is chemically, ${ }^{2,5}$ spectroscopically, ${ }^{2,3}$ and crystallographically $^{6}$ identical and isostructural to its synthetic phase, $\beta$-hematin. The magnetic susceptibility measurements and Mössbauer spectroscopy on $\beta$-hematin suggested the presence of a single highspin $(S=5 / 2)$ iron environment of largely axial symmetry in its bulk phase. ${ }^{3,7}$ Recently, the crystal structure of $\beta$-hematin was solved by X-ray powder diffraction. ${ }^{8}$ The structure is surprising in that rather than being a coordination polymer, as widely held before, ${ }^{2,3}$ it is a chain of dimers formed by the $\mathrm{Fe}^{\mathrm{III}}$ - protoporphyrin-IX molecules through reciprocal iron-carboxylate bonds to one of the propionic side chains of each porphyrin. The dimers then build chains linked by hydrogen bonds in the crystal. ${ }^{8}$

Despite the congruous nature of much of the spectroscopic data for the natural and synthetic phases, there is still considerable ambiguity in interpretation of the Electron Paramagnetic Resonance (EPR) results concerning the characterization of their local $\mathrm{Fe}$ environment. ${ }^{2,7,9-12}$ For example, previous work performed at conventional X-band $(9.5 \mathrm{GHz})$ frequency suggested a rhombic symmetry of the zero-field splitting (zfs) tensor, which was not in agreement with Mössbauer results. ${ }^{7}$ In this report, we present the definite conclusions of the spin state and properties of the ground state of hemozoin and $\beta$-hematin, using the power of multifrequency high-field EPR (HFEPR) to simplify and fully interpret spectra of the ferric ion. At the same time, we will use the HFEPR data to find correlations between structure and magnetic properties of both natural and synthetic malarial pigments.

Malarial pigment (hemozoin) was isolated from a K-1 chloroquine-resistant strain of Plasmodium falciparum. The pigment was isolated from late-stage trophozoites following nonproteolytic methods. ${ }^{14}$ Homogeneous single-phase microcrystalline powders of synthetic $\beta$-hematin were prepared by anhydrous dehydrohalogenation of hemin. ${ }^{15}$ The structure of a building block of the malarial pigment, $\left(\mathrm{Fe}^{\mathrm{III}} \text { - protoporphyrin-IX) }\right)_{2}$ dimer, 1, has a five-coordinate

\footnotetext{
$\S$ Polish Academy of Sciences.

† École Polytechnique Fédérale de Lausanne.

\#lorida State University.

"II McGill University.
}

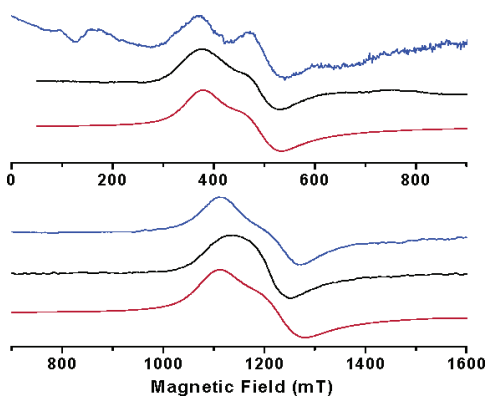

Figure 1. The low-field (high $g_{\text {eff }}$ ) turning point in the EPR spectra of hemozoin (blue) and $\beta$-hematin (black) at 34 (top) and $94 \mathrm{GHz}$ (bottom) at $10 \mathrm{~K}$. The red traces are powder-pattern simulations using the following spin Hamiltonian parameters: $|D|=5.80 \mathrm{~cm}^{-1},|E|=0.20 \mathrm{~cm}^{-1}, g_{\perp}=$ 1.90 (top) and $|D|=5.85 \mathrm{~cm}^{-1},|E|=0.075 \mathrm{~cm}^{-1}, g_{\perp}=1.95$ (bottom).

high-spin $\mathrm{Fe}^{\mathrm{III}}, S=5 / 2$, which is located $0.47 \AA$ out of the plane of the porphyrin and forms a relative short bond, $1.889 \AA$, with one of the oxygens of the protoporphyrin-IX propionic acid substituents. ${ }^{13}$ The distance between the two Fe ions within the dimer is 9.05(1) A, with a mean porphyrin plane separation of $4.44 \AA$. Due to their offsets, the nearest atoms in the porphyrin rings are separated by $5.00(1) \AA$, but the nearest iron neighbors are in adjacent unit cells where the $\mathrm{Fe}-\mathrm{Fe}$ separations are 7.86(1), 8.04(1), and 8.07(1) ̊..

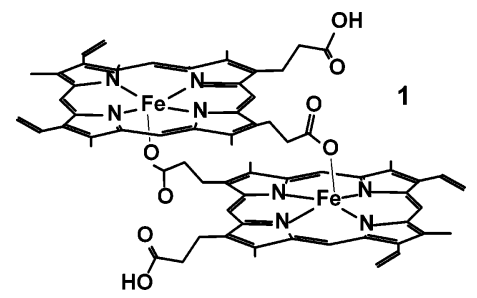

EPR spectra of hemozoin were acquired at cryogenic temperatures on two Bruker Elexsys spectrometers equipped with $\mathrm{TE}_{011}$ resonators: Q-band $(34 \mathrm{GHz})$ at the EPFL, and W-band $(94 \mathrm{GHz})$ at the NHMFL. $\beta$-Hematin was investigated in a wide range of frequencies $(27-500 \mathrm{GHz})$ in transmission-type single-pass spectrometers at the NHMFL. ${ }^{16}$ At each frequency, the $\beta$-hematin spectrum consists of a strong turning point at high effective $g_{\text {eff }} \sim$ 4.3-5.5, depending on frequency, and a weak turning point at $g_{\text {eff }}$ $=2$. At low frequencies $(27-94 \mathrm{GHz})$, the high $g_{\text {eff }}$ absorption line is partly split into two components (Figure 1). In this frequency range, the spectra of hemozoin are nearly identical to those of $\beta$-hematin. At higher frequencies, the splitting disappears, and a single line is observed (Figure S1 in the Supporting Information).

At high frequencies (above ca. $270 \mathrm{GHz}$ ), a new turning point is detected in the $\beta$-hematin spectra (Figure S1), appearing at yet 


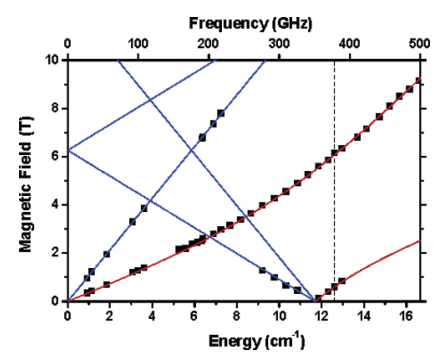

Figure 2. Resonance field versus quantum energy (or frequency) dependence of turning points in the powdered sample of $\beta$-hematin. Experimental points are marked by squares. Below $100 \mathrm{GHz}$, the average position of the partly split low-field resonance was taken. Lines were simulated using bestfitted spin Hamiltonian parameters as in text. Blue lines, parallel turning points; red lines, perpendicular turning points. For clarity, only those transition branches are plotted that are actually observed experimentally. The broken line corresponds to the frequency $(378.2 \mathrm{GHz})$ at which the bottom trace in Figure S1 was recorded.

lower fields than the previously described high $g_{\text {eff }}$ transition. The new signal approaches zero field at ca. $350 \mathrm{GHz}$ and starts increasing in field again at higher frequencies. Figure 2 summarizes all the resonances observed in $\beta$-hematin as a function of EPR quantum energy (or frequency).

The HFEPR spectra obtained for hemozoin and $\beta$-hematin can only be interpreted as originating from high-spin $\mathrm{Fe}^{\mathrm{III}}(S=5 / 2)$ described by a nearly axial spin Hamiltonian of the standard form, comprising both Zeeman and zfs terms, $H=\beta B \mathbf{g} S+D\left[S_{z}{ }^{2}-S(S\right.$ $+1) / 3]+E\left(S_{x}^{2}-S_{y}^{2}\right)$. An observation of the zero-field transition at $350 \mathrm{GHz}$, which, in the axial case, is equal to $2|D|$, yields the value of $|D|=5.83 \mathrm{~cm}^{-1}$. To our best knowledge, this is the first successful EPR detection of an inter-Kramers transition in a hemelike molecule. A complete set of intrinsic spin Hamiltonian parameters is delivered through a simultaneous fit to all the observed resonances, as previously described: ${ }^{17} D=+5.85(1) \mathrm{cm}^{-1}, E=0$, $g_{\perp}=1.95(1), g_{\|}=2.00(1)$ (the actual positive sign of $D$ is given by simulations of single-frequency spectra shown in Figure S1). The splitting in the perpendicular turning point of the intra-Kramers transition at low frequencies indicates, however, that the zfs tensor may not be entirely axial, as suggested by HFEPR. A fit to the Q-band spectra, where the observed splitting is the largest, yields the $|E|$ value of $0.2 \mathrm{~cm}^{-1}$, that is, a rhombicity of the $\mathrm{zfs}$ tensor $|E / D|$ equal to 0.035 . This is in very good agreement with the previous Mössbauer spectroscopy conclusions. ${ }^{7}$ The effect of the splitting decreasing and finally vanishing at higher frequencies is, however, puzzling since simulations show that it should increase rather than decrease. We see spin exchange as a tentative explanation of this phenomenon (see below).

In general, however, our results show that magnetic exchange within each dimer is negligible, even weaker than suggested by previous susceptibility measurements. ${ }^{3}$ Otherwise, the ground spin state of the dimer would be zero or an integer number, and not, as observed by us, $S=5 / 2$. Apparently, the significant distance between the two Fe centers and the number of chemical bonds between them make magnetic exchange very inefficient.

The spin Hamiltonian parameters obtained for the first time with high accuracy for a heme-like system are within the range observed in similar mononuclear Fe centers. It is known that the zfs parameter $D$ in five-coordinated $\mathrm{Fe}$ (III) complexes depends on the nature of the axial ligand. Thus, $D$ as measured by us is very similar in value to that determined for Fe in protoporphyrin-IX dimethyl ester with a fluoride axial ligand $\left(5.0 \mathrm{~cm}^{-1}\right)$ as measured by far-IR magnetic spectroscopy. ${ }^{18}$ A more relevant comparison can be made with another porphyrinic $\mathrm{Fe}$ (III) complex, with Fe axially ligated by an acetate residue, whereas $D$ was determined by Mössbauer spec- troscopy as $7.5 \mathrm{~cm}^{-1} .{ }^{19}$ As for the small but measurable rhombic parameter $E$, its presence was detected both in $\mathrm{Fe}(\mathrm{DmePP}-\mathrm{IX})$ $\left(\mathrm{N}_{3}\right)^{18}$ and in the above-mentioned complex with an axial acetate group, ${ }^{19}$ and apparently depends on the symmetry of the ligand itself. The propionate linker between the two porphyrins in $\beta$-hematin may be conducive to producing such a small rhombic distortion. An explanation of the effect of averaging of the $E$ value with increasing EPR frequency remains outside the scope of the present work, but we mention here the analogy with averaging the dipolar broadening of EPR resonances in solids with increasing observation frequency in the intermediate exchange regime. ${ }^{20}$ This analogy seems to be particularly attractive since the averaged perpendicular turning point at high frequencies actually gets narrower with increasing frequency and field in the solid, unlike the situation existing in a magnetically diluted system, such as myoglobin in frozen solution. ${ }^{21}$

Acknowledgment. This work was partially supported by the Polish KBN Grant \#2-PO3B-090-19, by the IHP-Contracts HPRICT-2001-00140, by G1MA-CI-2002-4017 (CEPHEUS) of the European Commission (A.S.), by the Burroughs-Wellcome Fund (P.W.S. and D.S.B.), by a CIHR Traineeship grant (C.G.), and by the Swiss National Science Foundation (B.V. and L.F.). We thank Dr. A. Ozarowski (NHMFL) for his simulation software, and Peter W. Stephens for his valuable assistance.

Supporting Information Available: EPR spectra of $\beta$-hematin in the high-frequency regime at $T=10 \mathrm{~K}$ are shown in Figure S1 (1 page, print PDF). This material is available free of charge via the Internet at http://pubs.asc.org.

\section{References}

(1) World Health Organization, The World Malaria Report 2005, www.who.int/malaria.

(2) Slater, A. F. G.; Swiggard, W. J.; Orton, B. R.; Flitter, W. D.; Goldberg, D. E.; Cerami, A.; Henderson, G. B. Proc. Natl. Acad. Sci. U.S.A. 1991 $88,325-329$.

(3) Bohle, D. S.; Conklin, B. J.; Cox, D.; Madsen, S. K.; Paulson, S.; Stephens, P. W.; Yee, G. T. In Inorganic and Organometallic Polymers II, Advanced Materials and Intermediates; Wisian-Neilson, P., Allcock, H. R., Wynne, K. J., Eds.; American Chemical Society: Washington, DC, 1994; pp 497515

(4) Dorn, A.; Stoffel, R.; Matile, H.; Bubendorf, A.; Ridley, R. G. Nature 1995, 374, 269-271.

(5) Fitch, C. D.; Kanjananggulpan, P. J. Biol. Chem. 1987, 262, 1555215555 .

(6) Bohle, D. S.; Dinnebier, R. E.; Madsen, S. K.; Stephens, P. W. J. Biol. Chem. 1997, 272, 713-716

(7) Bohle, D. S.; Debrunner, P.; Jordan, P. A.; Madsen, S. K.; Schultz, C. E. J. Am. Chem. Soc. 1998, 120, 8255-8256.

(8) Pagola, S.; Stephens, P. W.; Bohle, D. S.; Kosar, A. D.; Madsen, S. K. Nature 2000, 404, 307-310.

(9) Schoffa, G. Nature 1964, 203, 640-641.

(10) Arese, P.; Schwarzer, E. Ann. Trop. Med. Parasitol. 1997, 91, 501-516.

(11) Cammack, R.; Patil, D. S.; Linstead, D. J. Chem. Soc., Fraday Trans. 1994, 90, 3409-3410.

(12) Bremard, C.; Girerd, J. J.; Kowalewski, P.; Merlin, J. C.; Moreau, S. Appl. Spectrosc. 1993, 47, 1837-1842.

(13) Bohle, D. S.; Kosar, A. D.; Stephens, P. W. Acta Crystallogr. 2002, D58, $1752-1756$.

(14) Ashong, J. O.; Blench, I. P.; Warhurst, D. C. Trans. R. Soc. Trop. Med. Hyg. 1989, 83, 167-172.

(15) Bohle, D. S.; Helms, J. B. Biochem. Biophys. Res. Commun. 1993, 193 504-508.

(16) (a) Hassan, A. K.; Pardi, L. A.; Krzystek, J.; Sienkiewicz, A.; Goy, P.; Rohrer, M.; Brunel, L.-C. J. Magn. Reson. 2000, 142, 300-312. (b) Zvyagin, S. A.; Krzystek, J.; van Loosdrecht, P. H. M.; Dhalenne, G.; Revcolevschi, A. Physica B 2004, 346-347, 1-5.

(17) Krzystek, J.; Zvyagin, S. A.; Ozarowski, A.; Trofimenko, S.; Telser, J. J. Magn. Reson. 2006, 178, 174-183.

(18) Brackett, G. C.; Richards, P. L.; Caughey, W. S. J. Chem. Phys. 1971 54, 4383-4401.

(19) Bominaar, E. L.; Ding, X. Q.; Gismelseed, A.; Bill, E.; Winkler, H.; Trautwein, A. X.; Nasri, H.; Fischer, J.; Weiss, R. Inorg. Chem. 1992, $31,1845-1854$

(20) Krzystek, J.; Sienkiewicz, A.; Pardi, L.; Brunel, L. C. J. Magn. Reson 1997, 125, 207-211

(21) van Kan, P. J. M.; van der Horst, E.; Reijerse, E. J.; van Bentum, P. J. M.; Hagen, W. R. J. Chem. Soc., Faraday Trans. 1998, 94, 2975-2978. JA058420H 